\title{
The combination of different orthodontic treatment protocols and surgery techniques required in the management of patients born with cleft lip and/or palate: 10 years review
}

\begin{abstract}
Objectives: To summarize the combination of different Orthodontic treatment protocols and different Surgery techniques required in the management of patients born with cleft lip and/or palate.

Materials and methods: We searched the relevant articles from PubMed, on the web of science, EMBASE, Google scholar and manually search by using the following search terms: "Orthodontic management and cleft lip or palate", "Post orthodontic management and cleft lip or palate" "surgery techniques and cleft lip or palate" all relevant articles in English language published from 2007 to 2017 were reviewed Abstracts and title were evaluated and full-text analysis was performed, we excluded abstracts without full articles, articles published not in English language, Case reports, and all types of review.
\end{abstract}

Results: A total of 8 articles were included in the qualitative synthesis, they were of retrospective and cohort study design. Four articles1-4 assessed the surgical timing of cleft repair and favored early (mixed dentition) over late (permanent dentition) Secondary Alveolar bone grafting (SABG). The studies on postoperative orthodontic treatment5-8 and concluded that maxillary expansion and surgical advancement are generally needed for most cleft palate patients.

Conclusion: Orthodontic treatment plays an integral role in the rehabilitation phase toward the preparations of alveolar bone grafting and orthognathic surgery.

Keywords: Secondary Alveolar bone grafting, Distraction osteogenesis, Orthodontic treatment, cleft lip and or palate
Volume 9 Issue 3 - 2018

Iddi Suleiman Iddi,' Ferdinand Mabula Machibya, ${ }^{2}$ Liwei Xiao,' Zeng Yunting' 'Department of Dentistry, The Second Xiangya Hospital, Central South University, China \& Department of Dentistry, Mnazi Mmoja Referral Hospital,Tanzania

${ }^{2}$ Department of Orthodontics, Pedodontics and Community Dentistry, Muhimbili National Hospital (MNH), Tanzania

Correspondence: Liwei Xiao, Associate Professor of orthodontics in the Department of Dentistry, The Second Xiangya Hospital, Central South University, P.O.Box 41001 I, Changsha, Hunan, P.R China, Tel +86|3786|69806, Email xiao_Iw@csu.edu.cn

Received: June 02, 2018 | Published: June 12, 2018
Abbreviations: DO, Distraction osteogenesis; IMC, The Institute of Mother and child in Warsaw; RPE, Rapid Palatal Expander; SPE, Slow Palatal Expander; SABG, Secondary alveolar bone grafting; UCLP, Unilateral Cleft lip and Palate

\section{Introduction}

Cleft lip and cleft palate are the most common oral and facial malformations that occur in a very early stage of intrauterine life. ${ }^{9}$ Cleft lip and/or palate can be complete or incomplete, unilateral or bilateral. ${ }^{10}$ Brendt LE et al reported that cleft palate occurs around $7^{\text {th }}$ to $12^{\text {th }}$ week of gestation period while cleft lip and cleft alveolar occur in $4^{\text {th }}-8^{\text {th }}$ week of embryonic life. ${ }^{11}$ The management of oral cleft are often complex and expensive, which also require the presence of multidisciplinary team including oral and maxillofacial surgeons, Ear nose throat (ENT surgeons), plastic surgeons, orthodontist, speech therapist, nutritionist, psychologist, and dentist and specialized cleft nurses. ${ }^{10,12}$ Members of the cleft team should be aware to the economic status of the parents, psychosocial, linguistic and all factors that may affect the relationship between the primary caregivers of cleft infants or patient and other cleft team members. ${ }^{13,14}$ Most authors reported that orthodontic treatment and every stapes of surgical procedures should be well informed to the parents or primary caregivers of the cleft patient, doing so will help them to have clear understanding about all operational risk factors, treatment benefits, treatment costs, and duration of the treatment, parents or patients should also involve in making treatment planning this will help the parents or patients to know the short-term and long-term outcomes of the treatment for the satisfaction and psycho-social well-being of the parents and patient. ${ }^{14-16}$ Success of any type orthognathic surgery or alveolar bone grafting depends on proper orthodontic preparation, so surgeon and orthodontist should have same treatment objectives, and same languages, before surgical procedures orthodontist should correct malocclusions, monitoring tooth eruption, ensure good preparation of maxillary and mandibular arches, and develop of a proper plan for orthodontic tooth movement before and after surgery. ${ }^{17}$ The orthodontist should spend about 4 to 6 months to treat a cleft patient in preparation future alveolar bone grafting because any type of bone grafting without orthodontic preparation may lead to regrettable results associated with posterior cross bite, severe maxillary arch constriction and may cause unnecessary secondary surgical repair. ${ }^{18}$

Concerning postsurgical treatment goals unfortunately there is no universal principle and agreement between surgeon and orthodontist, although all surgeon and orthodontist have same guiding principle that the jaws or teeth should be moved to permit the maxilla and mandible 
to be in a desired and aesthetically position but each country has their own principle on how to approach and to solve cleft problems. ${ }^{18,19}$ It has been reported that the postsurgical orthodontic treatment of patients born with cleft lip and/or palate may involve four common stages.

1. Orthodontic treatment during the period of primary dentition.

2. Orthodontic treatment during the period of mixed dentition.

3. Orthodontic treatment during the period of permanent dentition.

4. Orthodontic treatment after growth completion. ${ }^{19,20}$

In the world literature exists many controversies $\mathrm{s}$ about surgical timing and techniques of post-orthodontic procedures in the management and treatment of cleft lip and/or palate, therefore the aim of this review is to create summary and comparisons of the combination of different Orthodontic treatment protocols and different Surgery techniques performed in the management of patients born with cleft lip and/or palate.

\section{Materials and methods}

\section{Data sources}

We retrieved articles by using the following predetermined terms with various combination and modifications "orthodontic management and cleft lip or palate", "Post orthodontic management and cleft lip or palate" "surgery techniques and cleft lip or palate" articles retrieved from PubMed, web of science, EMBASE and by manual search, all articles published from Jan 2007 to November 2017 regarding the combination of different orthodontic treatment protocols and surgery techniques required in the management of patients born with cleft lip and/or palate were screened, participants were humans born with cleft lip and/or palate, any relevant work published in English language which contains required information was included in the review, abstracts and title were evaluated for compliance with the inclusion criteria and full-text analysis were performed then all publication in English language with matching eligible criteria were included. We excluded abstracts without full text, articles published not in English languages, Case reports, and all types of review.

\section{Results}

\section{Surgical treatment}

\section{Early cleft repair}

Article SN 1 Camille et al., ${ }^{1}$ described the early surgical treatment outcome and recommended cleft surgery at neonate age fight against infanticide and avoid the rejection of children particularly in the African background as well as providing relief to the family and social rehabilitation benefits for the child (Table 1). ${ }^{1}$

\section{Secondary Alveolar bone grafting Timing}

Articles SN 2 to 4 in the table above reported about alveolar ridge grafting timing

Walia et al. ${ }^{3}$ The age of the patient should be within the mixed dentition period. Meyer \& Mølsted. ${ }^{2}$ Successful SABG performed before the eruption of the tooth distal to the cleft. Mean age in the success group was 12.1 Rawashdeh et al. ${ }^{4}$ Early (before maxillary canine eruption) unilateral SABG had a success rate of $90 \%$ while late (after the maxillary canine eruption) unilateral SABG rate was $80 \%$ (Table 1$).^{2-4}$

\section{Postoperative orthodontic treatment}

\section{For successful teeth alignment SN 5}

Seike et al., ${ }^{5}$ assessed the evaluating the grafted alveolar cleft to predict the orthodontic treatment outcome and concluded that successful orthodontic treatment required marginal bone level greater than $3 \mathrm{~mm}$, and vertical height of more than $6 \mathrm{~mm}$ together with anteroposterior bone width greater than $5 \mathrm{~mm}$, as well as bone mineral density of $<350 \mathrm{mg} \mathrm{Ca}_{5}\left(\mathrm{PO}_{4}\right) \mathrm{OH} / \mathrm{ML}$ (Table 1). ${ }^{5}$

\section{Orthodontic Maxillary expansion and surgical \\ SN 6 to 8 reported about maxillary expansion}

Lisson et al., ${ }^{6}$ concluded that patients with either unilateral or bilateral cleft palate experience sagittal growth inhibition of the maxilla after primary surgery and maxillary advancement after completion of growth are equally needed in both cleft types. Vasant et al., ${ }^{7}$ compared the effectiveness of Quad helix and conventional rapid maxillary expansion appliances and found that Quad helix was as effective as conventional rapid maxillary expansion appliances among cleft patients. Oberoi et al., ${ }^{8}$ found that multiple missing maxillary teeth, secondary palate procedures including pharyngeal flaps and inconsistent team care with delayed orthodontic intervention are the factors contributing to maxillary hypoplasia that needs maxillary advancement surgery in individuals with nonsyndromic unilateral cleft lip and palate (UCLP) (Table 1). ${ }^{6-8}$

\section{Discussion}

\section{Introduction}

Early management of oral cleft may bring relief to the family, enhance social rehabilitation of the child and self-esteem of the parents (Table 1). ${ }^{1}$ The presurgical orthodontic treatment offer conducive environment for the surgical and non-surgical procedures to be conducted under required conditions. ${ }^{21}$ Orthodontist should make sure that cleft patient has proper teeth arrangement before orthognathic surgery. The technique of Le Fort I distraction osteogenesis enhances the formation of callous bone in a gap so it greatly lowers the soft tissue restriction and reduces relapse rate. ${ }^{22}$ Combination of surgical and orthodontic treatment in the period of primary dentition (6months to 7 years). This is also called the first phase of post-surgical orthodontic treatment, it called so because it takes place after cleft lip repair which is supposed to be done at the age between 3 and 5 months, ${ }^{15}$ some authors reported that the cleft lip repair is performed during 6 to 8 weeks of age..$^{22}$ but in case of bilateral cleft lip and alveolus Balint Nemes et al reported the definitive closure of bilateral cleft lips conducted when the patients were aged 6 months. ${ }^{23}$ In primary dentition some authors reported the necessity of guidance of the eruption of deciduous teeth (milk teeth) ${ }^{15}$ while other authors the acquisition and enhancement of speech development. ${ }^{19}$ Contrary to Dorota Cudzilo insisted that the main treatment goals during primary dentition is to achieve a symmetrical positioning of maxillary teeth, doing so may favor bone grafting to be done under required condition, no removable orthodontic appliance required before bone grafting but $3-4$ months after surgery removable orthodontic appliance may be used. ${ }^{18}$

The primary bone grafting is performed in primary dentition from 0 month to 2 years of age, and the alveolar bone cleft repair may be conducted simultaneously with cleft lip or cleft palate repair but early secondary repair is started after the full eruption of primary dentition 
which is between 2 to 6 years. ${ }^{23}$ The main objective of primary bone grafting is to promote closure of primary palatal cleft, to elevate alar floor that may help in lateral tissue growth and to close nasobuccal or per alveolar fistula at an earlier stage. Combination of surgical and orthodontic treatment during mixed dentition (7-12 years). Secondary alveolar bone grafting (SABG) advocated by Boyne et al in 1972, it has been reported that (SABG) may be performed at any stage and time after the primary lip and palate repair ${ }^{24}$ but good success of alveolar bone grafting occurs when it is performed before the eruption of the maxillary canine on the cleft side. ${ }^{2}$

Based on timing SABG can be divided into three main groups.

1. Early secondary alveolar bone grafting performed in primary dentition between 2 to 6 years.

2. Intermediate (transitional) SABG applied during mixed dentition between 6 and 12years.

3. Late (Tertiary) SABG conducted in permanent dentition after an eruption of all permanent teeth 12years and above.

This phase needs close collaboration between the surgeon and orthodontist, doing so will help to determine the anatomical limits of Presurgical expansion because overexpansion of maxilla may create fistula of the oronasal region. ${ }^{19}$ Some authors suggested that the main purpose of this stage is to create enough space for accommodation of permanent teeth and ensure good development of the upper jaw in all three dimension. ${ }^{18}$ but others authors suggested that among main concerns of Orthodontist in this phase is monitoring eruption of permanent teeth. ${ }^{15}$ Shetye et al., ${ }^{19}$ highlighted that orthodontist could use removable orthodontic appliance to promote the growth of the jaws and preparation of the maxillary arch before SABG and all discrepancies in maxillary arch form or width should be corrected before secondary alveolar bone graft, the alveolar bone graft surgery could be performed between 8-9 years of age. ${ }^{15}$ Takuya Seike et al., mentioned that the main goals of alveolar bone grafting for proper orthodontic treatment are

a. Stabilization of maxilla

b. Facilitate eruption of permanent teeth

c. Allow easy movement of neighboring teeth and closing of oronasal fistula (Table 1). ${ }^{5}$

A cohort study done by Meyer et al., ${ }^{2}$ followed more than 10years, concluded that $82 \%$ of 123 secondary alveolar bone grafts were a long-term surgical success but the timing of secondary alveolar bone grafting is critical in regard to the age of the patient and the stage of tooth eruption immediately distal to the cleft side. ${ }^{2}$ Regarding surgical timing related to maxillary canine, it exists a minor controversies between authors, Wirthlin ${ }^{24}$ reported that intermediate secondary alveolar bone grafting performed when the unerupted canine has $1 / 2$ to $2 / 3$ rds of its root formation, ${ }^{24}$ while other authors only highlighted that the best stage for SABG when the canine adjusted to the cleft has completed $1 / 2$ to $3 / 4$ of its root formation. ${ }^{25}$ Jorg A Lisson et al., ${ }^{6}$ reported that the inhibition of maxillary growth in growing patient having complete bilateral and unilateral cleft lip, alveolus and palate occur after completion of primary surgery so it is necessary to consider surgical maxillary advancement after completion of a growth spurt in both types of clefts (Table 1). ${ }^{6}$ Many authors recommended that full fixed orthodontic appliances should be conducted after maxillary expansion which is generally performed at age of 10 years and 7 months and Presurgical maxillary expansion is generally performed after secondary alveolar bone graft $\mathrm{f}^{20,26}$ but if cleft children had mid face deficiency or negative overjet (class III mal occlusion) the protraction of the maxillary segment by using face mask or head gear, mini implants (mini screws) or mini plates which provide an orthodontic force that can be used to correct maxillary segment to be in proper position is indicated and in order to provide better conditions for future bone grafting the maxillary expansion should be conducted at age of 9years 11 months, class III elastics may be used to correct the overjet. ${ }^{19,26}$ In case of a severe maxillary reduction in Dorota et al recommended the use of thick-wire appliances such as expander and combination of a Delaire or Petite type face mask to reduce sagittal displacement of maxilla. ${ }^{18}$ Concerning maxillary expansion in cleft children many authors recommended the use of rapid palatal expander (RPE) and slow palatal expander (SPE), ${ }^{7,17}$ it has been reported that both slow expander(Quad helix) and RPE were capable to correct posterior crossbites in cleft patients but slow expander such as Quad helix represented more advantages in the ability of correcting molar rotation than rapid expander(Hyrax) but unfortunately both of them were not showed significant results in increasing intercanine and molar width expansion(Table 1). ${ }^{7}$ Raj M Vyas et al., ${ }^{17}$ suggested the use of a new device, in case cleft patient present maxillary hypoplasia rapid palatal expander using a borne-borne distraction device may be used, this type of expander is capable to increase palate surface area by a mean of $28.9 \mathrm{~mm} 2$ inter molar distance up to $8.4 \mathrm{~mm}$ and intercanine increased by an average of $9.5 \mathrm{~mm}$, the main advantage of this expander relapse rate is very slow is between $4 \%$ and $7 \%$ for cleft patients. ${ }^{17}$ Regarding the timing of orthodontic treatment of cleft lip and/or palate in mixed dentition, it exists a controversy between authors, Alexander reported that fixed orthodontic treatment should begin after alveolar bone grafting or post-Alveolar bone Graft development which generally occurs at age $8-12$ years Old but when the facial appearance of the cleft child is among of the major concern and there is no good improvement in maxillary growth early/interceptive maxillary distraction osteogenesis(DO) may be considered but the protraction face mask should be not used unless is planned to work after distraction osteogenesis, ${ }^{20}$ contrary to other authors supported the use of face mask, head gear even before distraction osteogenesis, ${ }^{19,26} \mathrm{John}$ reported that many cleft palate centers perform maxillary expansion with or without fixed orthodontic appliance before alveolar bone grafting. ${ }^{24}$ Combination of surgical and orthodontic treatment during the period of permanent dentition (12 years to adulthood). In this phase the orthodontic treatment is generally conducted by fixed orthodontic appliances, the treatment may be focused on correction of the position of permanent teeth, achieving a correct occlusal relationship and achieving a proper position of alveolar process of the upper and lower jaw. ${ }^{18}$ In a conventional orthodontics which is generally conducted between 11-15years old, the extraction in the lower arch may reduce crowding and the retroclination of the incisors, may also enhance the relationship between lower and upper arch and in order to avoid relapse of anterior cross bite especially after mandibular growth, the orthodontic retention for the patient with a bony alveolar cleft needs to be permanent reinforced with both removable and fixed techniques. ${ }^{20}$ The management of cleft in permanent dentition may be categorized into three groups:

\section{Orthodontic treatment of cleft patients with no skeletal deformity}

Shetye et al., ${ }^{19}$ reported that in isolated cleft lip and cleft alveolus or cleft only on the soft palate the management of malocclusion is approximately same as non-cleft patient so they will be treated by using fixed orthodontic alone, the correction of mild dental anterior or 
posterior cross bites can be treated before finishing growth spurt by advancing arch wire and for posterior cross bites may be treated by Quad helix or with arch wire expansion. ${ }^{19}$ It has been reported that in treating cross bites in cleft patients, maxillary expansion by using Quad helix provide satisfactory results than rapid maxillary expanders. ${ }^{7}$ Shapira et al., ${ }^{27}$ reported that $77 \%$ of a nonsyndromic cleft lip and palate patient present Hypodontia and $74 \%$ of maxillary permanent lateral incisors were missed, smaller dental arch contributed by a large number of missing teeth. ${ }^{27}$

\section{Orthodontic treatment of cleft patients with mild skeletal deformity}

In case cleft patients observed with minimal esthetics concerns and have mild skeletal discrepancy orthodontic treatment with dental compensation will take place. ${ }^{19}$ If cleft patient present cross bite in the anterior segment, the maxillary expanders may be used, many orthodontist prefer to use slow maxillary expander because it has the ability to expand the jaw interiorly than posteriorly ${ }^{7}$ but some practitioners prefer rapid maxillary expanders (RPE) because once the appliance is cemented there is no need for the appliance for activation and some authors think that RPE create more skeletal expansion than dental expansion. ${ }^{24}$

\section{Orthodontic treatment of cleft patients having a severe skeletal discrepancy}

The combination of surgical and orthodontic techniques offers satisfactory results in terms of aesthetics and functional. ${ }^{19}$ When the skeletal discrepancy is very clear and the growth spurt is not yet complete, Cudziło ${ }^{18}$ recommended beginning the distraction osteogenesis procedure of the maxilla which has the aim of advancement of the upper jaw which promotes the adaptations of the surrounding tissue, ${ }^{18}$ some authors suggested that in severe skeletal discrepancy, in order to achieve optimal occlusion the cleft patient may require either only maxillary protraction or maxillary protraction and mandibular set back to compensate the jaws discrepancy. ${ }^{19}$

Table I Summary of the study objectives and outcomes/conclusion of the articles reported orthodontic preparation before and after surgical procedures of oral clefts

\begin{tabular}{lll}
\hline SN Author & Objective & Conclusion and recommendation \\
\hline Surgical Treatment timing &
\end{tabular}

\section{Surgical Treatment timing}

I Camille et al.

Meyer \& Mølsted

To assess the long-term outcome of secondary
alveolar bone grafting (SABG) in cleft lip and palate patients and to examine relationships between preoperative and postoperative factors and overall long-term bone graft success.

To assess the outcome of Secondary alveolar bone

$4 \quad$ Rawashdeh et al. ${ }^{4}$ grafting (SABG) before (early $S A B G$ ) and after (late $S A B G$ ) the eruption of the permanent maxillary canine in unilateral and bilateral Jordanian cleft subjects.
I. Neonatal surgery has advantages in fighting against infanticide and avoid the rejection of those children particularly in the African background

2. It brings a relief to the family and social rehabilitation benefits for the child.

I. Long-term follow-up is required to achieve maximum advantage of secondary alveolar grafting.

2. The age of the patient should be within the mixed dentition period, irrespective of sex, socio-economic status.

I. The timing of secondary alveolar bone grafting is critical with regard to the age of the patient and the stage of eruption of the tooth distal to the cleft.

a. It was found that the success rate was significantly lower if SABG was performed after eruption of the tooth dista to the cleft.

b. Mean age in the success group was 12.1 years and 13.6years in the failure group

I. Early unilateral SABG had a success rate of $90 \%$ while late unilateral SABG rate was $80 \%$.

2. The overall success rate of the unilateral SABG was higher than the rate for the bilateral cases.

Postoperative orthodontic treatment

5 Seike et al..$^{5}$

Predicting the orthodontic treatment outcome by evaluating the grafted alveolar cleft

To determine the extent of maxillary deficiency at

6 Lisson et al. ${ }^{6}$ an early stage during the transitory dentition, and to identify factors that might indicate the need for a later maxillary advancement.
Successful orthodontic treatment required marginal bone level $>3$ $\mathrm{mm}$, vertical height $>6.5 \mathrm{~mm}$, anteroposterior bone width $>5 \mathrm{~mm}$, and bone mineral density<350mg $\mathrm{Ca}_{5}\left(\mathrm{PO}_{4}\right) \mathrm{OH} / \mathrm{mL}$.

I. Patients with complete UCLP and BCLP experience sagittal growth inhibition of the maxilla after primary surgery.

2. A later need for maxillary advancement after completion of growth occurs equally in both cleft types.

3. It is impossible to predict a need for later maxillary osteotomy during the transitory dentition. 
Table Continued
Author
Objective
Conclusion and recommendation

Surgical Treatment timing

Vasant et al. ${ }^{7}$

Oberoi et al. ${ }^{8}$

To delineate factors that may contribute to maxillary hypoplasia requiring maxillary advancement surgery in individuals with nonsyndromic unilateral cleft lip and palate (UCLP)
Maxillary expansion using the Quad helix appliance represents a reasonable alternative to using conventional rapid maxillary expansion appliances among cleft patients.

I. Maxillary hypoplasia that will require a Le Fort I advancement can be determined as early as age 10

2. Contributing factors to maxillary underdevelopment are:

a. Multiple missing maxillary teeth

b. Secondary palate procedures including pharyngeal flaps

c. Inconsistent team care with delayed orthodontic intervention.

\section{Conclusion}

Orthodontic treatment plays an integral role in the rehabilitation phase toward preparations of alveolar bone grafting and orthognathic surgery. In case the cleft patient presenting severe skeletal discrepancy the combination of fixed orthodontic treatment and orthognathic surgery are the best solutions. Secondary Alveolar Bonne Grafting is likely to be successful when performed early mixed dentition than late permanent dentition. Most of the unilateral and bilateral cleft palates patients need maxillary advancement after completion of their growth and among common factors contributed to the occurrence of maxillary under development are multiple missing maxillary teeth, delayed orthodontic intervention, inconsistent team care, and secondary palate surgical procedures.

\section{Acknowledgements}

None.

\section{Author's contributions}

ISI, FMM, LWX, and ZY contributed to the design and searching appropriate articles for the review. ISI and FMM did the correction of the review. All authors contributed equally to the preparation of the review, revised the review and approved the final version.

\section{Conflict of interest}

The author declares that there is no conflict of interest.

\section{References}

1. Camille A, Evelyne AK, Martial AE. Advantages of early managemen of facial clefts in Africa. International Journal of Pediatric Otorhinolaryngology. 2014;78(3):504-506.

2. Meyer S, Mølsted K. Long-term outcome of secondary alveolar bone grafting in cleft lip and palate patients: A 10-year follow-up cohort study. Journal of Plastic Surgery and Hand Surgery. 2012;47(6):503-508.

3. Walia A. Secondary alveolar bone grafting in cleft of the lip and palate patients. Contemporary Clinical Dentistry. 2011;2(3):146-154.

4. Rawashdeh MA, Al Nimri KS. Outcome of secondary alveolar bone grafting before and after eruption of the canine in Jordanian patients with cleft lip and palate. Journal of Craniofacial Surgery. 2007;18(1):331-
1337.

5. Seike T, Hashimoto I, Matsumoto K. et al. Early postoperative evaluation of secondary bone grafting into the alveolar cleft and its effects on subsequent orthodontic treatment. The Journal of Medical Investigation. 2012;59(1,2):152-165.

6. Lisson JA, Weyrich C. Extent of maxillary deficiency in patients with complete UCLP and BCLP. Head and Face Medicine. 2014;10(1):1-7.

7. Vasant MR, Menon S, Kannan S. Maxillary expansion in cleft lip and palate using quad helix and rapid palatal expansion screw. Medical Journal Armed Forces India. 2009;65(2):150-153.

8. Oberoi S, Chigurupati R, Vargervik K. Morphologic and Managemen Characteristics of Individuals With Unilateral Cleft Lip and Palate Who Required Maxillary Advancement. Cleft Palate Craniofac J. 2000;45(1):42-9.

9. Li W, Wang S, Zhang Y. Relationships among satisfaction, treatment motivation, and expectations in orthodontic patients: A prospective cohort study. Patient Preference and Adherence. 2016;10:443-447.

10. Antoun JS, Fowler PV, Jack HC. Oral health-related quality of life changes in standard, cleft, and surgery patients after orthodontic treatment. American Journal of Orthodontics and Dentofacial Orthopedics. 2015;148(4):568-575.

11. Jayashree M, Paul S. Naso alveolar molding in early management of cleft lip and palate. Journal of Indian Prosthodontist Society 2013;13(3):362-365.

12. Balkaya MC, Sultan H, Erdem S. Prosthetic rehabilitation of a patient with a unilateral cleft palate: A clinical report. The Journal of Prosthetic Dentistry. 2014;111(4):269-272.

13. Kasten EF, Schmidt SP, Zickler CF, et al. Team Care of the Patient with Cleft Lip and Palate. Current Problems in Pediatric and Adolescent Health Care. 2008;38(5):138-158.

14. Larson BE. Orthodontic preparation for orthognathic surgery. Oral and Maxillofacial Surgery Clinics of North America. 2014;26(4):441-458.

15. Adeyemi TA. Cleft Lip and Palate Care in Nigeria. Journal of Craniofacial Surgery. 2015;26(4):1106-1108.

16. Li HX, Luo MY, Luo JY. et al. A discriminant analysis prediction model of non-syndromic cleft lip with or without cleft palate based on risk factors. BMC Pregnancy and Childbirth. 2016;16(1):368.

17. Vyas RM, Jarrahy R, Sisodia M. Bone-borne palatal distraction to correct

Citation: Iddi IS, Machibya FM, Xiao L, et al.The combination of different orthodontic treatment protocols and surgery techniques required in the management of patients born with cleft lip and/or palate: I0 years review. J Dent Health Oral Disord Ther. 20I8;9(3): I94-199. 
the constricted cleft maxilla. J Craniofac Surg. 2009;20(3):733-736.

18. Cudziło D. Orthodontic treatment of patients with a cleft lip and palatestandard procedures at the Warsaw Institute of Mother and Child. Developmental Period Medicine. 2014;XVIII(1):53-58.

19. Shetye PR. Update on treatment of patients with cleft-Timing of orthodontics and surgery. Seminars in Orthodontics. 2016;22(1): 45-51.

20. Cash AC. Orthodontic treatment in the management of cleft lip and palate. Front Oral Biol. 2012;16:111-123.

21. Nemes B, Fábián G, Nagy K. Management of prominent premaxilla in bilateral cleft lip and alveolus. Cleft Palate-Craniofacial Journal. 2013;50(6):744-746.

22. Silveira A Da, De Moura PM, Harshbarger RJ. Orthodontic considerations for maxillary distraction osteogenesis in growing patients with cleft lip and palate using internal distractors. Semin Plast Surg. 2014;28(4):207-211.
23. Daw JL, Patel PK. Management of alveolar clefts. Clinics in Plastic Surgery. 2004;41(2):303-313.

24. Wirthlin JO. The orthodontist's role in the management of patients with cleft lip and palate undergoing alveolar bone grafting. Seminars in Orthodontics. 2017;23(3):268-278.

25. Williams J. The recognition and management of isolated cleft palate. Community Pract. 2012;85(10):28-31.

26. Rocha R, Ritter DE, Locks A. Ideal treatment protocol for cleft lip and palate patient from mixed to permanent dentition. American Journal of Orthodontics and Dentofacial Orthopedics. 2012;141(4 Suppl):140-148.

27. Shapira Y, Lubit E, Kuftinec MM. Hypodontia in Children with Various Types of Clefts. Angle Orthodontist. 2000;70(1):16-21. 